\title{
The way forward for TB vaccines
}

Tuberculosis (TB) is now the largest single-pathogen cause of global adult mortality ${ }^{1}$; more than 10 million incident cases of disease occur worldwide every year. In 2018, global TB prevention, diagnosis and treatment efforts were estimated to cost US $\$ 10.4$ billion ${ }^{2}$. With such a high global burden, and a current average annual incidence rate decline of only $1.8 \%$, new efficacious vaccines are urgently needed to accelerate progress towards the World Health Organization 'End TB' and elimination goals ${ }^{1}$.

The year 2018 was particularly uplifting for the field of TB vaccine development, as unprecedented results were reported from two proof of concept efficacy trials. Van Der Meeren and colleagues reported that the GSK subunit vaccine M72/AS01 ${ }_{\mathrm{E}}$, consisting of $M t b$ antigens and monophosphoryl lipid A and QS21 formulated in liposomes as adjuvant, prevented pulmonary TB disease in adults already-infected with Mycobacterium tuberculosis (Mtb) ${ }^{3}$. The vaccine efficacy in this phase IIB trial was $54 \%(95 \% \mathrm{Cl}, 3-78)$. Nemes and colleagues reported that bacille Calmette-Guérin (BCG) revaccination of previouslyuninfected adolescents protected against $M t b$ infection, as defined by sustained conversion of the Quantiferon Gold In Tube test ${ }^{4}$. The BCG revaccination vaccine efficacy was $45 \%$ $(95 \% \mathrm{Cl}, 6-68)$ for this secondary study endpoint.

These results could translate into a significant breakthrough for TB prevention if the protective effects against pulmonary disease could be confirmed (for M72/AS01E) or demonstrated (for BCG revaccination), and shown to be generalisable. For example, in India, preliminary mathematical modelling has suggested that a vaccine rolled out in 2025 with characteristics similar to those demonstrated for M72/AS01E in the phase IIB trial, and with a duration of efficacy of 10 years, could, as a single intervention, reduce TB incidence in 2050 by around $40 \%{ }^{5}$.

For M72/AS01 $\mathrm{E}$, a larger confirmatory trial, powered to improve the accuracy of efficacy estimates, is now needed. This trial could also explore whether age and gender affect protection, as suggested in secondary analyses based on a limited number of cases ${ }^{3}$. Further investigations should also include examining protection in Mtb-uninfected persons and in high risk groups such as HIV-infected persons, and on what the duration of vaccine protection could be. Involving multiple geographies would be required to support generalisability of the findings. Investments are not only needed for the next phase of clinical trials, but also to ensure sustainable and affordable supply of vaccine antigens and adjuvant. Preparatory work on licensure and policy pathways, equitable access, and delivery should be initiated early to allow rapid implementation, should phase III trial results be positive.

The observation that adolescent BCG revaccination may prevent sustained $M t b$ infection also requires further investigation, for multiple reasons. The estimate of effect should be generated in trials with more precision, as the confidence intervals were wide ${ }^{4}$. Then, whether prevention of $M t b$ infection translates into protection against TB disease should be characterized, as ultimately a policy recommendation for adolescent BCG revaccination by the WHO would likely require demonstration of prevention of pulmonary TB disease ${ }^{6}$. At this stage, evidence appraisal would need to take into account previous studies of BCG revaccination showing little, or no, impact on prevention of tuberculosis disease ${ }^{7-10}$. Even if 
confirmed, obstacles to delivery of BCG would include the current contra-indication of the vaccine in HIV-infected persons.

Multiple other TB vaccine candidates are currently in clinical development, mostly for prevention of adolescent/adult TB disease. Some candidates are being developed for BCG replacement in neonates, although this strategy is likely to have lower population level impact before 2050, than efficacious vaccination at older ages ${ }^{11}$. Three vaccines have entered, or are about to enter, efficacy trials for prevention of adult TB disease: two inactivated nontuberculous mycobacterial vaccines, Vaccae (Anhui Zhifei Longcom, contains Mycobacterium vaccae) and Immunovac (Cadila Pharmaceuticals, contains Mycobacterium $w$, aka $M$. indices pranii), and a recombinant BCG vaccine (VPM-1002, Serum Institute India, contains a listeriolysin insertion and urease deletion). Three vaccines will be tested for ability to prevent recurrence of adult TB disease after successful cure: VPM-1002 and 2 novel subunit vaccines, ID-93/GLA-SE (IDRI, contains two Mtb antigens and glucopyranosyl lipid formulated in a stable emulsion as adjuvant) and H56/IC31 (States Serum Institut, contains three $M t b$ antigens and an 11-mer antibacterial peptide and a synthetic oligodeoxynucleotide as adjuvant). Vaccines that are currently being, or will soon be, tested for ability to prevent Mtb infection include DAR-901 (Dartmouth, contains inactivated Mycobacterium obuense) and ID-93. Prevention of infection trials have the advantage of requiring relatively small sample sizes, but then require prevention of disease confirmation. Multiple other viral vectored, subunit and whole cell vaccine candidates are in earlier clinical trials, including a recombinant Mtb, MTBVAC (Biofabri), which has two mutations rendering the organism at least as safe as BCG in animal models.

Among preclinical advances in 2018, a recombinant, attenuated cytomegalovirus (CMV) vaccine which expresses 6-9 Mtb antigens demonstrated the best protection against pulmonary $M t b$ challenge ever reported in non-human primates ${ }^{12}$. This vector induces immunity in this animal model regardless of pre-existing CMV exposure, and may have a mechanism of action that is distinct from most other TB vaccination approaches, by inducing effector T cells that are widely distributed in all organs, including the lung, and which are continuously replenished due to ongoing antigen expression (most $\mathrm{T}$ cell targeted vaccines induce a long-lived central memory $T$ cell response). Human testing of this candidate is under intense preparation.

The TB vaccine field needs to move forward with a sense of urgency, and in a strategic, data-driven way. The current funding available for TB vaccines ${ }^{13}$ will be insufficient to drive rapid progress, and therefore an increase and diversification of resources is required. Some of these resources should be used to increase clinical trial capacity, and to continue the discovery and advanced development of early pipeline candidates.

A better understanding of protective immunity against TB is critical. Toward this goal, samples available from the two recent efficacy trials ${ }^{3,4}$ could now be examined to identify mechanisms and correlates of protection, which, in turn, may contribute to accelerate clinical testing of candidates and guide future vaccine discovery.

Models of vaccine use-case, in diverse geographical settings, should be developed to inform vaccine development strategies that could deliver most impact. Further modelling and health-economic research, into the public health impact and value of TB vaccines would 
support rational prioritisation of investments. Last, but no means least, we need to prepare for success; prompt implementation of successful vaccination strategies will be critical to help prevent the more than 4000 deaths due to TB each and every day.

The opinions expressed herein are those of the authors and do not necessarily reflect the views and decisions of the World Health Organization or the Bill and Melinda Gates Foundation. RGW is funded the UK Medical Research Council (MRC) and the UK Department for International Development (DFID) under the MRC/DFID Concordat agreement that is also part of the EDCTP2 programme supported by the European Union (MR/P002404/1), the Bill and Melinda Gates Foundation (TB Modelling and Analysis Consortium: OPP1084276/OPP1135288, CORTIS: OPP1137034/OPP1151915, Vaccines: OPP1160830) and UNITAID (4214-LSHTM-Sept15; PO 8477-0-600).

\section{REFS}

1 World Health Organization. Global Tuberculosis Report 2018. http://apps.who.int/iris/bitstream/handle/10665/274453/9789241565646-.

2 Stop TB Partnership. The Global Plan to End TB 2016-2020. The Paradigm Shift. http://www.stoptb.org/assets/documents/global/plan/GlobalPlanToEndTB_TheParadigm Shift_2016-2020_StopTBPartnership.pdf.

3 Van Der Meeren O, Hatherill M, Nduba V, et al. Phase $2 \mathrm{~b}$ Controlled Trial of M72/AS01E Vaccine to Prevent Tuberculosis. N Engl J Med 2018; 379: 1621-34.

4 Nemes E, Geldenhuys H, Rozot V, et al. Prevention of M. tuberculosis Infection with H4:IC31 Vaccine or BCG Revaccination. N Engl J Med 2018; 379: 138-49.

5 Harris R. The potential epidemiological impact of new TB vaccines. 2018; published online Oct 23.

6 World Health Organization. WHO Preferred Product Characteristics for New Tuberculosis Vaccines. 2018.

7 Rodrigues LC, Pereira SM, Cunha SS, et al. Effect of BCG revaccination on incidence of tuberculosis in school-aged children in Brazil: the BCG-REVAC cluster-randomised trial. Lancet 2005; 366: 1290-5.

8 Randomised controlled trial of single BCG, repeated BCG, or combined BCG and killed Mycobacterium leprae vaccine for prevention of leprosy and tuberculosis in Malawi. Lancet 1996; 348: 17-24.

9 Leung CC, Tam CM, Chan SL, Chan-Yeung M, Chan CK, Chang KC. Efficacy of the BCG revaccination programme in a cohort given BCG vaccination at birth in Hong Kong. Int J Tuberc Lung Dis 2001; 5: 717-23.

10 Tala-Heikkilä MM, Tuominen JE, Tala EO. Bacillus Calmette-Guérin revaccination questionable with low tuberculosis incidence. Am J Respir Crit Care Med 1998; 157: 1324-7.

11 Harris RC, Sumner T, Knight GM, White RG. Systematic review of mathematical models exploring the epidemiological impact of future TB vaccines. Hum Vaccin Immunother 
2016; 12: 2813-32.

12 Hansen SG, Zak DE, Xu G, et al. Prevention of tuberculosis in rhesus macaques by a cytomegalovirus-based vaccine. Nat Med 2018; 24: 130-43.

13 Treatment Action Group. Tuberculosis Research Funding Trends 2005-2017. 2018. 
